\title{
Communicating ATLAS: adapting to an ever-changing media landscape
}

\author{
Ana María Rodríguez Vera* \\ York University \\ Toronto, Canada \\ E-mail: a.mariarv@cern.ch
}

Katarina Anthony-Kittelsen ${ }^{\dagger}$

Universita degli Studi di Udine

Udine, Italy

E-mail: katarina.anthony@cern.ch

\begin{abstract}
Communicating the status and achievements of the ATLAS Experiment at the LHC has been a core objective of the ATLAS Collaboration since its founding. To match an ever-changing media landscape, ATLAS has tailored its communication strategy to produce content that effectively targets key audiences. The comprehensive approach of ATLAS communications is explored, with a focus on strategic themes, effective distribution channels, and message. The success of this approach is examined and the effect on user experience is evaluated.
\end{abstract}

XXIX International Symposium on Lepton Photon Interactions at High Energies - LeptonPhoton2019 August 5-10, 2019

Toronto, Canada

* Speaker.

${ }^{\dagger}$ on behalf of the ATLAS Collaboration 


\section{Introduction}

Communication of scientific research, to both the scientific community and general public, is an essential component of the long-term success of any experiment. The ATLAS Collaboration has made a constant effort to communicate the status and achievements of this LHC experiment and adapt to an ever-changing media landscape [1]. In order to reach a larger audience and to produce content that has a more effective impact, the communication strategy has been tailored to fit the needs of the current media environment, where social media plays a key role. These proceedings focus solely on the public communications published on the ATLAS website and the different social media channels, and examines the success of each approach.

\section{The ATLAS website}

The primary source for communicating the ATLAS experiment's [2] most up-to-date status and achievements to the general public is the ATLAS public website [3]. The following is a list of the long-form types of content found therein, along with a short description of the scope of each one:

- News Articles and Press Statements: broad, non-expert audience with coverage driven by events.

- Physics Briefings: results-driven coverage for a higher-level, but still non-expert audience.

- Features: long-form feature articles on key physics subjects aimed at a broad, non-expert audience, with an educational purpose.

- Portraits: a series of interviews presenting collaborators whose contributions have helped shape the ATLAS experiment.

- Blog posts: written by members of the collaboration, giving personal perspectives on ATLAS.

\section{Maximizing the reach of ATLAS public content: a new approach}

The migration of media to the web over two decades ago changed the conditions of the way in which information is communicated. Interaction is more social and immediate, as well as more mobile-based. Science communication strategies need to adapt to this rapidly changing environment. The ATLAS Education and Outreach team has implemented a multi-fold approach for the creation and dispersion of content [4], maximizing the use of ATLAS' social media channels and taking advantage of new external platforms, while maintaining the ATLAS public website as the primary source of content.

\subsection{Engagement through social media}

Although the ATLAS website remains the main source of information on the ATLAS experiment, the collaboration also has accounts in the following social media platforms: Instagram [5], YouTube [6], Twitter [7] and Facebook [8]. These accounts not only redirect users to the ATLAS 
website, but also share social-media-specific content created by ATLAS Education and Outreach, in order to maximize engagement. Traditional communications, as the ones described in Section 2, are also posted on social media.

- Social media videos: length is kept between 1-2 minutes, with concise content and captions. The primary message of the video is delivered in less than three seconds, to capture the short attention span of users. Videos are 1:1 ratio, improving their visibility on mobile devices.

- Instagram: posts and stories used as a new platform for evergreen and unique short-form content (e.g., "Physicist Friday" interview series).

Figure 1 shows the twelve best performing videos published on ATLAS social media in the last twelve years. Those videos posted under the current ATLAS social content strategy are indicated with the word "new". The best performing video content was 2018 video "Listen to Heavy Ions collide in ATLAS", which was viewed 230,000 across three social media platforms [9].

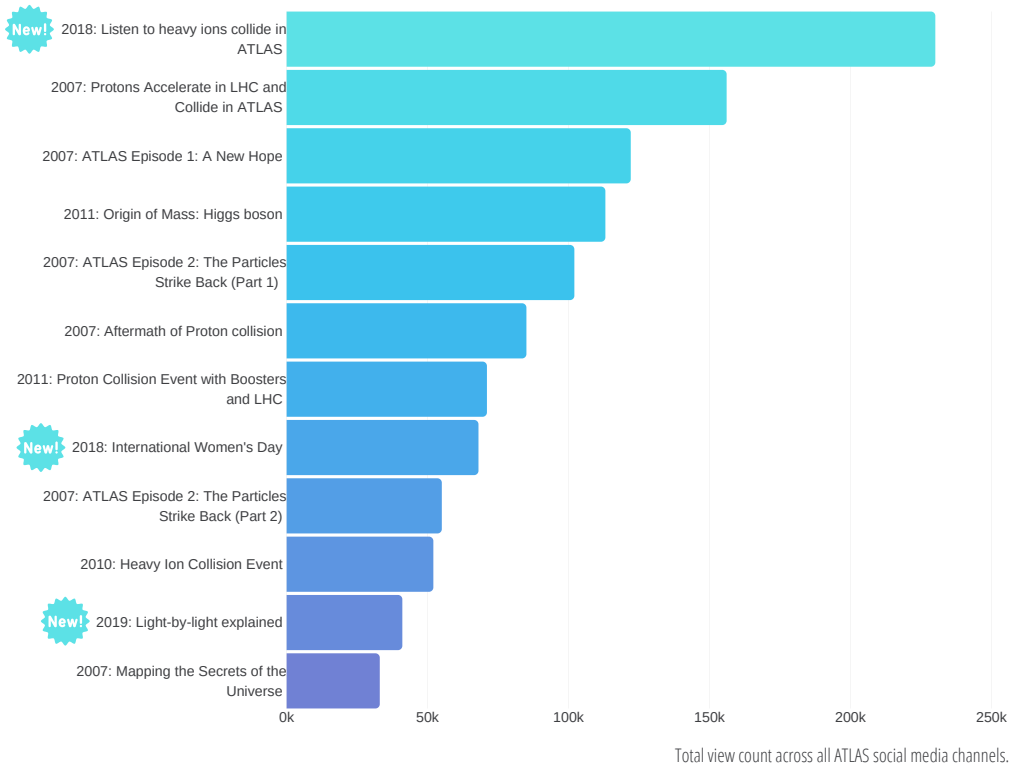

Figure 1: The twelve best performing videos published on ATLAS social media channels between 20082019. Highlighted "new" are the videos published since the implementation of the new ATLAS social content strategy.

\subsection{New platforms}

The ATLAS Education and Outreach team has included dissemination platforms that share long-form ATLAS updates with the audience. These platforms include:

- Phys.org: Physics Briefings published directly on the Phys.org news website, which has a large established user base.

- Facebook Notes: Physics Briefings and Press Releases directly on the social media platform - of particular value for mobile users who remain on the application. 
- Google News: Since 2017, all ATLAS updates appear in Google News. This has increased the overall visibility of ATLAS updates, while also ensuring that an official voice is represented amongst coverage of high-profile stories.

Currently, Phys.org accounts for $\sim 70 \%$ of all views of ATLAS Physics Briefings. Furthermore, the publication of physics briefings as Facebook Notes has produced an increase in the read-rate of $\sim 250 \%$. With the addition of ATLAS updates in Google News, referrals from this platform have been the third largest driver of traffic to the ATLAS website in 2018.

\section{Conclusions}

The ATLAS Education and Outreach team is constantly improving its strategy to communicate the status and results of the ATLAS experiment at the LHC. The new approach proposed and implemented includes the use of social media platforms, and has proven to be successful in reaching the public with content specifically tailored for this purpose. Finally, the strategy to continue promoting long-form content through both social media and new platforms that direct a wider public to the ATLAS official website, has proven to be successful.

\section{References}

[1] S. Goldfarb, et. al., Innovating science communication: the structure supporting ATLAS Education \& Outreach, Nuclear and Particle Physics Proceedings, 273-275 (2016) 1276. https://doi.org/10.1016/j.nuclphysbps.2015.09.204

[2] ATLAS Collaboration, The ATLAS Experiment at the CERN Large Hadron Collider, JINST 3 (2008) S08003.

[3] ATLAS Collaboration official website https://atlas.cern

[4] A. Sidoti, et. al., The Social Content Strategy of the ATLAS Collaboration, ATL-OREACH-PROC-2018-002 (2018)

[5] ATLAS Collaboration official Instagram account https://www.instagram.com/ATLASexperiment

[6] ATLAS Collaboration official YouTube channel https://www.youtube.com/ATLASexperiment

[7] ATLAS Collaboration official Twitter account https://twitter.com/ATLASexperiment

[8] ATLAS Collaboration official Facebook page https://www.facebook.com/ATLASexperiment

[9] ATLAS Collaboration "Listen to heavy ions collide in the \#LHC", November 22 2018, 11:21 AM GMT, Tweet. 